\title{
Minimizing Uncertainties Impact in Decision Making with an Applicability Study for Economic Power Dispatch
}
H Wang
S Wang
$\mathrm{R}$ Fan
Z F Zhang

\section{September 2016}

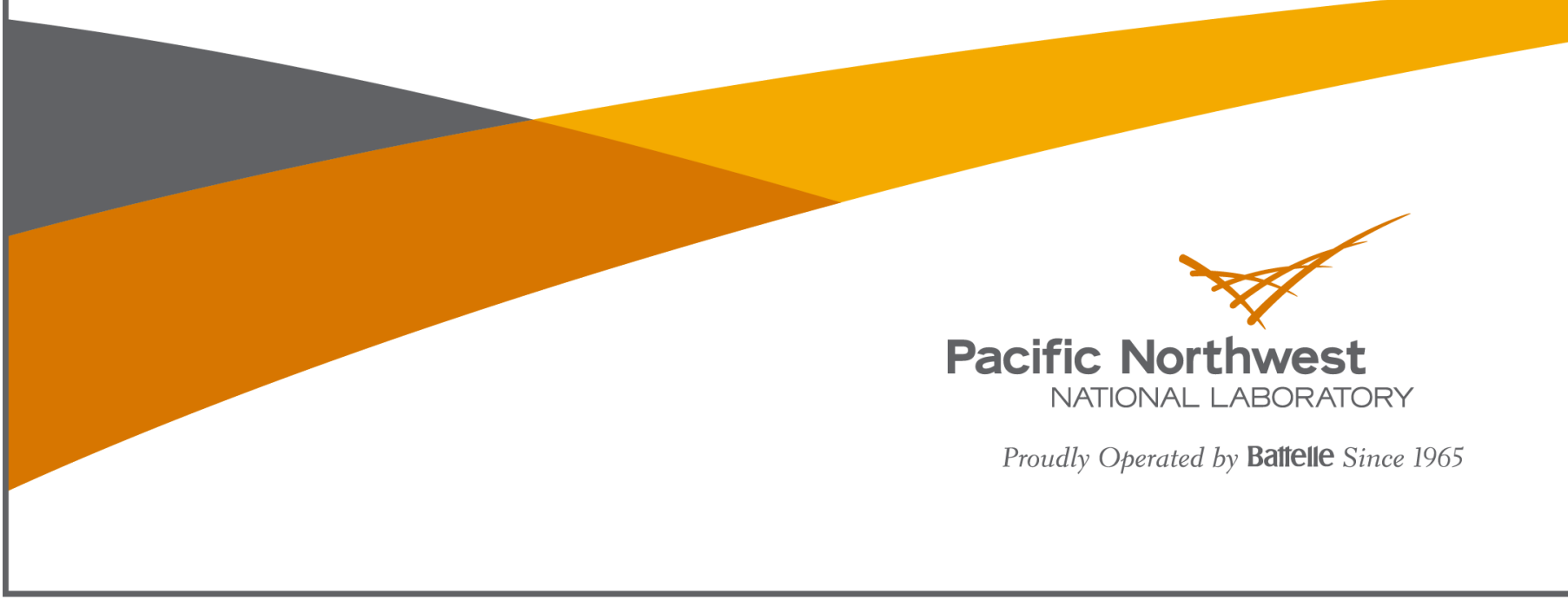




\title{
DISCLAIMER
}

This report was prepared as an account of work sponsored by an agency of the United States Government. Neither the United States Government nor any agency thereof, nor Battelle Memorial Institute, nor any of their employees, makes any warranty, express or implied, or assumes any legal liability or responsibility for the accu racy, completeness, or usefulness of any information, apparatus, product, or process disclosed, or represents that its use would not infringe privately owned rights. Reference herein to any specific commercial product, process, or service by trade name, trademark, manufacturer, or otherwise does not necessarily constitute or imply its endorsement, recommendation, or favoring by the United States Government or any agency thereof, or Battelle Memorial Institute. The views and opinions of authors expressed herein do not necessarily state or reflect those of the United States Government or any agency thereof.

\author{
PACIFIC NORTHWEST NATIONAL LABORATORY \\ operated by \\ BATTELLE \\ for the \\ UNITED STATES DEPARTMENT OF ENERGY \\ under Contract DE-AC05-76RL01830
}

Printed in the United States of America

Available to DOE and DOE contractors from the

Office of Scientific and Technical Information,

P.O. Box 62, Oak Ridge, TN 37831-0062;

ph: (865) 576-8401

fax: $(865) 576-5728$

email: reports@adonis.osti.gov

Available to the public from the National Technical Information Service

5301 Shawnee Rd., Alexandria, VA 22312

ph: (800) 553-NTIS (6847)

email: orders $a$ ntis.gov <http://www.ntis.gov/about/form.aspx>

Online ordering: http://www.ntis.gov

से This document was printed on recycled paper

(8/2010) 



\begin{abstract}
This report summaries the work performed under the LDRD project on the preliminary study on knowledge automation, where specific focus has been made on the investigation of the impact of uncertainties of human decision making onto the optimization of the process operation. At first the statistics on signals from the Brain-Computing Interface (BCI) is analyzed so as to obtain the uncertainties characterization of human operators during the decision making phase using the electroencephalogram (EEG) signals. This is then followed by the discussions of an architecture that reveals the equivalence between optimization and closed loop feedback control design, where it has been shown that all the optimization problems can be transferred into the control design problem for closed loop systems. This has led to a "closed loop" framework, where the structure of the decision making is shown to be subjected to both process disturbances and controller's uncertainties. The latter can well represent the uncertainties or randomness occurred during human decision making phase. As a result, a stochastic optimization problem has been formulated and a novel solution has been proposed using probability density function (PDF) shaping for both the cost function and the constraints using stochastic distribution control concept. A sufficient condition has been derived that guarantees the convergence of the optimal solution and discussions have been made for both the total probabilistic solution and chanced constrained optimization which have been well-studied in optimal power flows (OPF) area.
\end{abstract}

A simple case study has been carried out for the economic dispatch of powers for a grid system when there are distributed energy resources (DERs) in the system, and encouraging results have been obtained showing that a significant savings on the generation cost can be expected. 



\section{Contents}

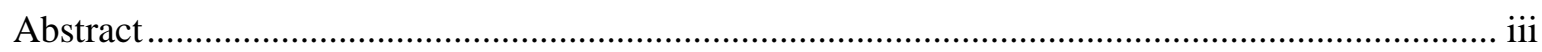

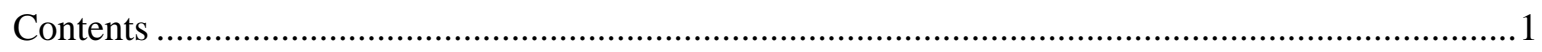

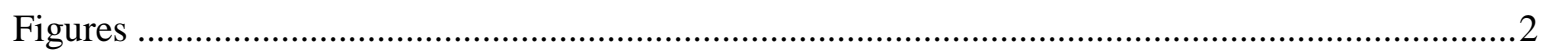

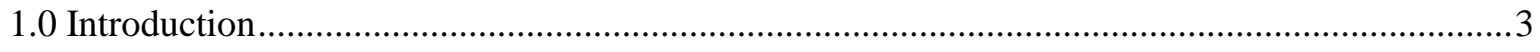

2.0 Formulation of Optimization as a Feedback Control Design Problem .....................................6

2.1 Optimization is a special case of feedback control design ............................................... 6

2.2 Source of uncertainties in decision making - system uncertainties vs humam

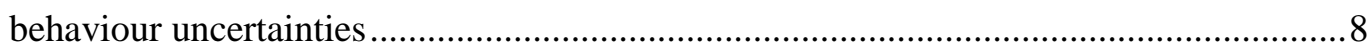

3.0 The Generalized Framework for Decision Making Using pdf Shaping Approach.................... 10

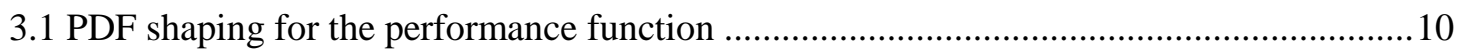

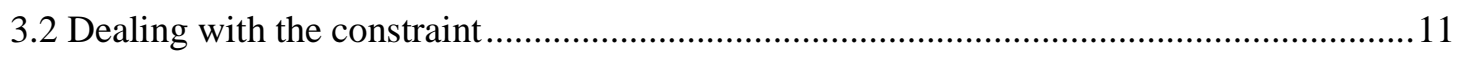

$\begin{array}{ll}3.3 \text { Total probabilistic solutions } & 13\end{array}$

3.4 Chanced constrained optimization - probability constraints $\quad 13$

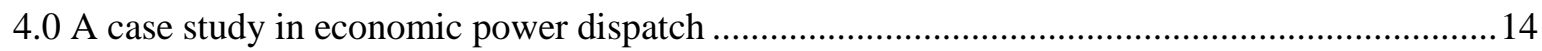

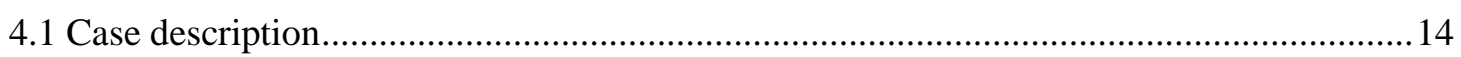

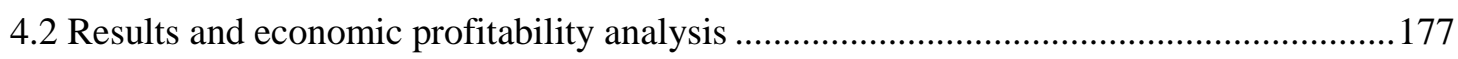

4.3 Switching strategy for whole horizon economic power dispatch.................... 19

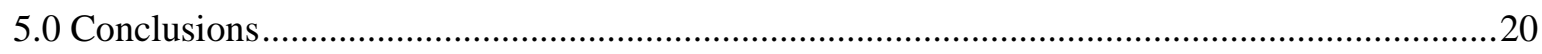

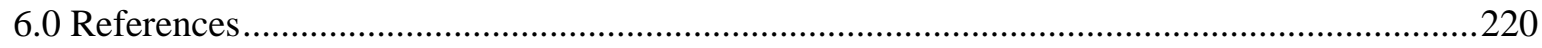




\section{Figures}

Figure 1. The 2D operational structure of complex industrial processes ......................................... 3

Figure 2. PDFs of EEG signals for experienced and non-experienced operators ([3]) .....................5

Figure 3. Treating the optimization as a closed loop feedback control problem ..............................

Figure 4. The optimization framework that incorporates uncertainties from both the process and

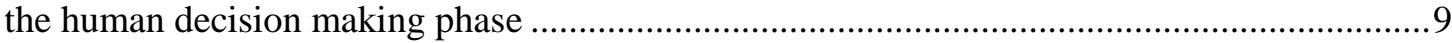

Figure 5. PDFs of the performance function before and after the optimization ............................. 10

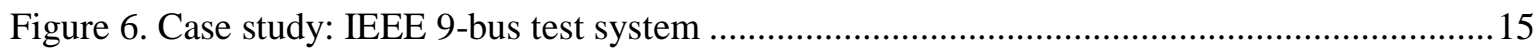

Figure 7. PDF-Shaping results on stochastic economic dispatch ............................................. 18

Figure 8. Optimization results of PDF: single objective VS multi-objective $\ldots \ldots \ldots \ldots \ldots \ldots \ldots \ldots$

Figure 9. Optimization of CDF: single objective VS multi-objective $\ldots \ldots \ldots \ldots \ldots \ldots \ldots \ldots \ldots \ldots \ldots$ 


\subsection{Introduction}

Operation of manufacturing systems involves raw material supplies chain, material processing lines and after-sales services. Examples are steel making, mineral processing, power systems, car manufacturing, papermaking and petro-chemical plants, where in the production phase there are a lot of control systems working collaboratively to fulfill the required production. These control systems have multiple layered interface with the on-site human operators at different level and the whole system works horizontally through material processing along production lines and vertically through the integration of different layers of operation control, planning and scheduling as well as operational management ([1]-[2]). Such a structure is shown in the following figure.

During the operation of these processes, the ultimate purpose is to achieve optimized product quality and production efficiency. This means that at least there are several performance indexes that need to be optimized at the same time, namely the product quality indexes, runnability and costs. This constitutes a multi-objective optimization problem. Indeed, it is well-known that once the production structure is fixed, it is the control systems that would play a vital role to realize the optimality of the operation. Assuming that the control loop layer has been well designed, the final stage of decision variables would be the set-points applied to these control loops along the concerned production line.

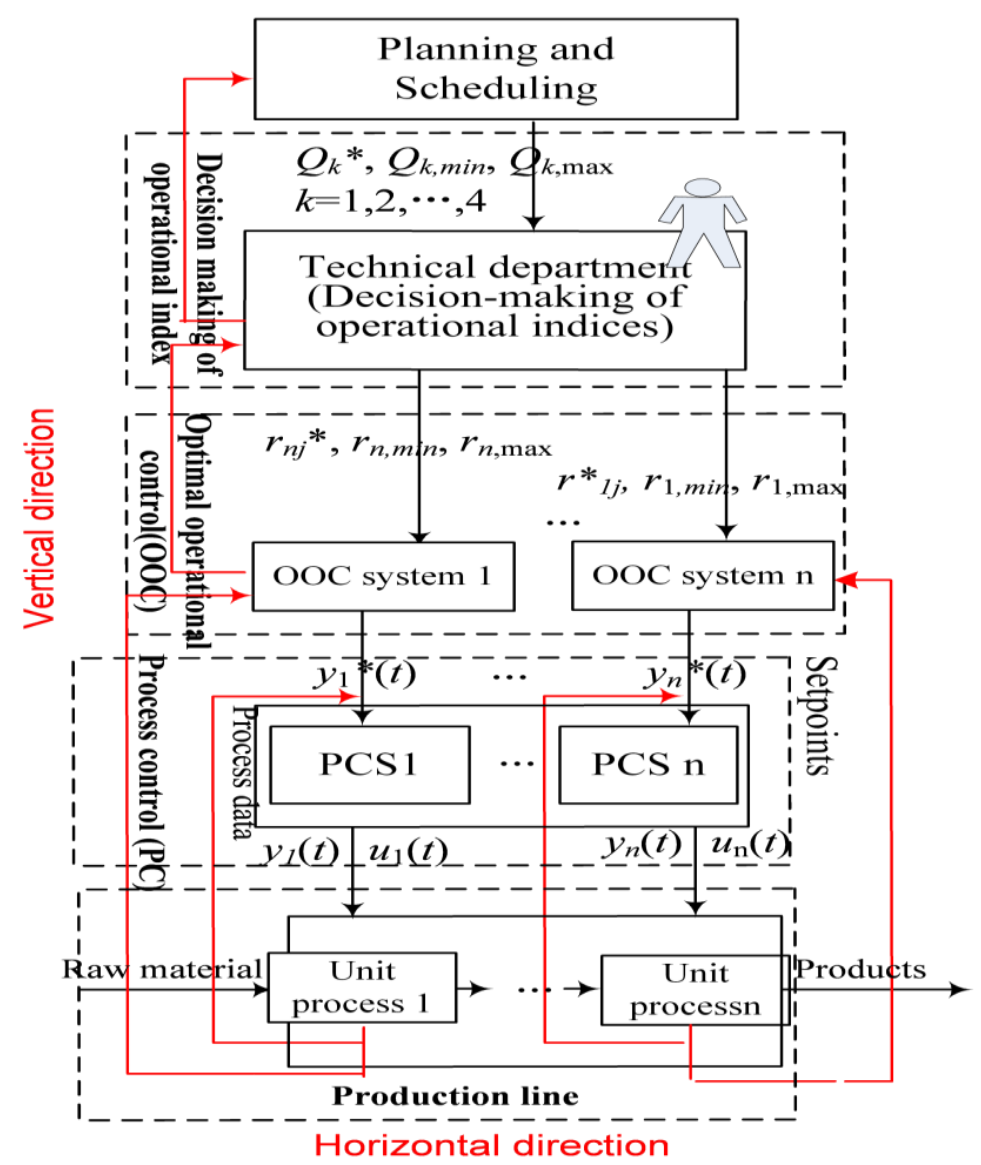

Figure 1. The 2D operational structure of complex industrial processes 
With the multiple layer structure as shown in Figure 1, optimization would generally take place in each layer and at different time scale. For example the optimization at the control loop layer would be realized in line with the closed loop sampling rate of the concerned closed loop system using the knowledge of the process, the operation control layer would require certain degree of the involvement of human operators who help to fine tune the set-points to the control loops whilst the optimization at the planning and scheduling level would require a longer time and involve significant assistance of human operators for the decision making. This presents new challenges for the optimization where human-in-loop aspects need to be considered, and in particular the effect of uncertainties of human operators in the decision making phase need to be quantitatively analyzed so as to assess the optimality effect and the related robustness of the optimal operation of the concerned complex systems when they are subjected to unexpected variations in either raw materials or the operational environment.

It is therefore imperative to access how the decision-making of human operator is integrated with the actual process information so as to optimize production performance in terms of enhanced product quality and reduced costs (e.g., raw materials and energy usage, and effluent discharge).

At present, the plant level operational control and management have been largely solved ([1-2]), where a group of manipulated (decision) process variables can be generally formulated from the models of the process to optimize the process operation together with the decisions made by the human operators. In general, the decision making phase of human operators contains uncertainties in the time-horizon in terms of observation, use of knowledge and decision realization. Since these uncertainties will affect the effect of the decision-making, there is a challenging issue here on how the negative effect of these uncertainties on the optimization can be minimized. This constitutes one of the key issues yet to be solved for the knowledge automation for industrial processes control which is a new concept nowadays following the launch of industrial 4.0 in Germany.

A preliminary study by Hong Wang [3] has shown that the uncertainties of human operator's decision making can be measured through EEG (brain) signals, where the brain signals of inexperienced operators would generally exhibit large portion of uncertainties and randomness which lead to a widely distributed probability density functions (pdf) as shown in Figure 2. It is therefore imperative that such a measure be used by well-defined process variables in the minimization of the impact of uncertainties embedded in human operator's decision making. This forms the main objective of the proposed study.

Following the completion of the PDM project where a comprehensive review of existing development on knowledge automation has been made, this proposed LDRD project has been focused on the development of a method on how the uncertainties in knowledge automation can be measured and used in operational optimization for process control. In particular, we are going to use methods from different disciplines including techniques from Brain Computer Interface (BCI), EEG signal processing, knowledge engineering, data mining, and decision science ([4]-[6]) to look into the following aspects in a logical order:

1) Investigate the ways to quantify knowledge quality and randomness using signals collected from the brain via EEG;

2) Develop a method on performance index modeling that can describe the relationships between uncertainties of human decision variables and the well-defined process variables.

3) Explore the framework that can be used to realize the risk-minimization of human decision making by adjusting a group of well-defined process variables. 

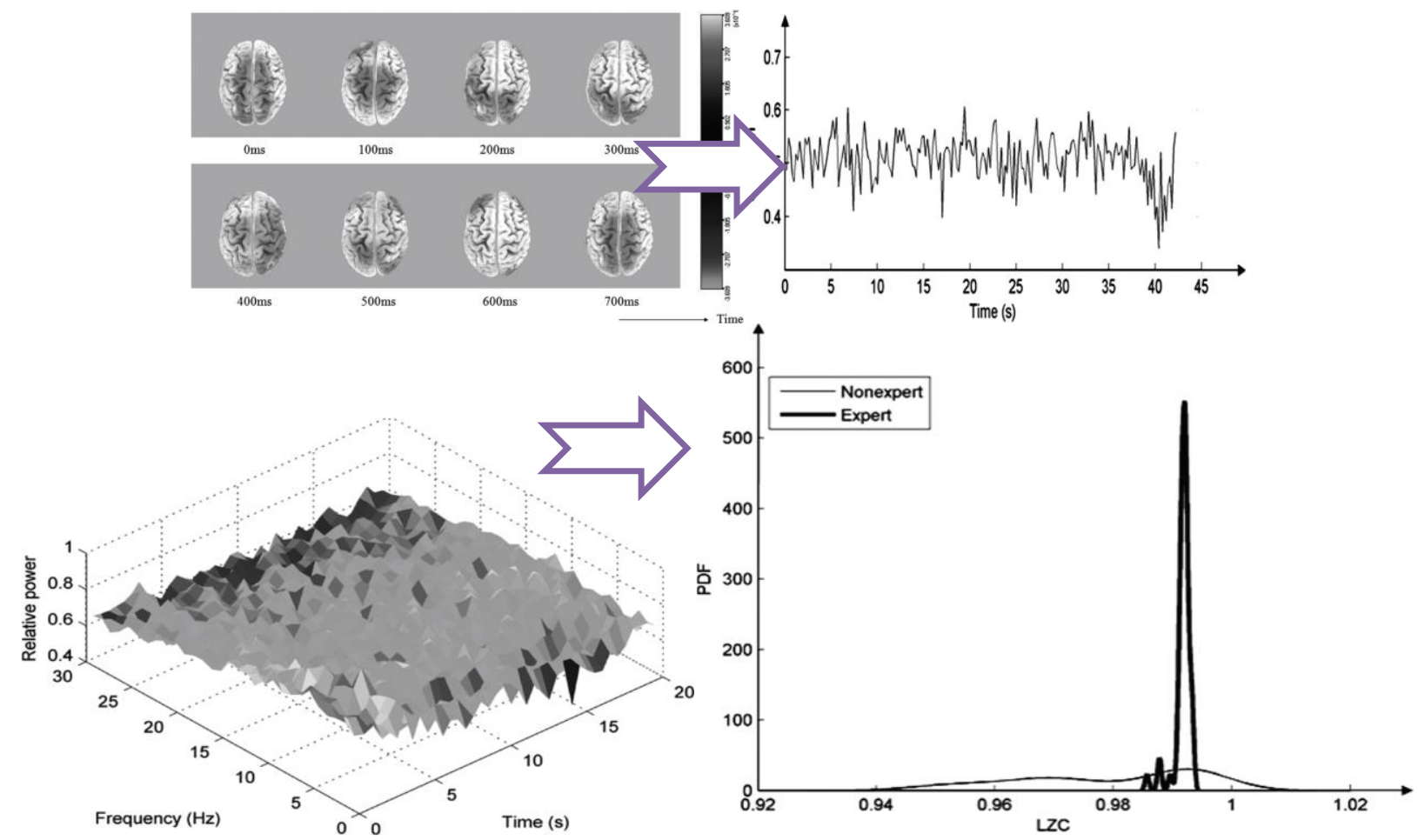

Figure 2. PDFs of EEG signals for experienced and non-experienced operators ([3])

In line with the above tasks, the following Milestones will be envisaged.

- Select relevant context and define use-cases for BCI-based knowledge automation. (September 2016)

- Find a robust method or strategy that can be used to quantify quality and randomness of knowledge extracted from human brain (September 2016)

The first milestone has been achieved where a set of BCI based information has been collected and analyzed in order to define the uncertainties treatment to be described later. The second milestone has also been achieved where a novel stochastic optimization solution is obtained that uses probability density function (pdf) as a means to quantify the randomness of the knowledge extraction in the optimization phase and shows how such a pdf can be used to design optimization algorithms. These will be described in details in the following sections.

In specific, a novel sufficient condition has been derived that guarantees that the decision sequence converges to a crisp value in solving the stochastic optimization problem that are subjected to both decisionmaking uncertainties and process disturbances. 


\subsection{Formulation of Optimization as a Feedback Control Design Problem}

Optimization has been a subject of study for many years and is widely required in many areas. In general a simple optimization is formulated as solving the following problem

$$
\begin{aligned}
& \min _{x} J(x) \\
& \text { s.t. } f(x)=0
\end{aligned}
$$

where $J$ in (1) is a performance function to be optimized and $x$ is a vector that groups a set of decision variables to realize such an optimization. In the optimization $x$ should also satisfy the constraints defined by the second equation (see (2)).

\subsection{Optimization is a special case of feedback control design}

Without the loss of generality it is assumed that $J$ is always positive. In this context, the purpose of the optimization can be interpreted as to select an optimal value of $x$ so that $J$ is made as close as possible to zero. Since in many cases the actual optimization algorithm is realized in a form of recursion for $x$, if we denote $x_{k}$ as the value of $x$ at sample number $k$, then the following recursive optimization algorithm is generally used

$$
x_{k}=\pi\left(x_{k-1}, J\left(x_{k-1}\right), \theta\right)
$$

where $\pi(., .,$.$) is a functional operator that represents the designed optimization algorithm and \theta$ is a group of learning rates. For example when there is no constraint and $J$ is differentiable and is defined on a compact set, the well-known gradient descent algorithm that minimizes $J$ recursively is given by

$$
x_{k}=x_{k-1}-\theta \frac{\partial J\left(x_{k-1}\right)}{\partial x_{k-1}} \quad k=1,2, \ldots
$$

This means that the functional operator of the gradient descent optimization algorithm expressed in equation (4) is given by

$$
\pi\left(x_{k-1}, J\left(x_{k-1}\right), \theta\right)=x_{k-1}-\theta \frac{\partial J\left(x_{k-1}\right)}{\partial x}
$$

Taking the optimization algorithm as a "control" algorithm, this means that to optimize $J$ subjected to the required constraints one needs to design a "controller" that can ensure ideally that the following tracking error convergence

$$
\lim _{\boldsymbol{k} \rightarrow+\infty} \boldsymbol{e}_{\boldsymbol{k}}=\lim _{k \rightarrow+\infty}\left[0-J\left(x_{k}\right)\right]=0
$$

By taking $J$ as the output of the "plant" to be controlled and $x$ as the control input to be applied to the "plant", the following "closed loop control system" can be readily obtained. 


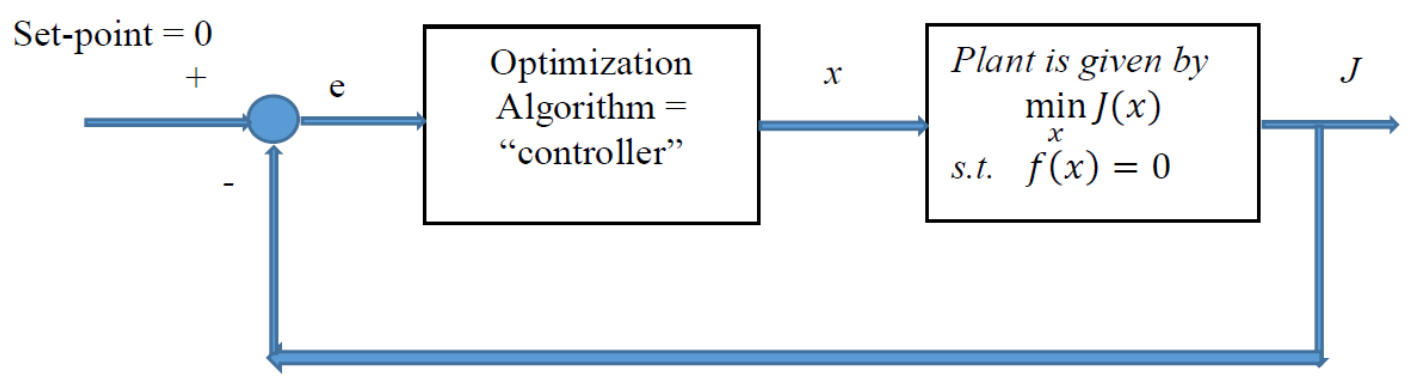

Figure 3. Treating the optimization as a closed loop feedback control problem

Since $J$ is always positive and the set-point to the above closed loop control system is always zero, the optimization can be regarded as a special case of the well-known feedback control system design. This reveals a very important fact which says that any control design methods can be considered as a possible candidate in the design of an optimization algorithm. This fact has therefore broadened the scope of the optimization and at least the following statements are true,

- Multi-objective optimization can be treat as a multi-input and multi-output (MIMO) controller design problem;

- The rather rich stability and robustness analysis tools (such as Lyapunov stability theory) developed in control theory can be directly employed to analyze the performance of the concerned optimization. For example, the convergence and dynamic performance of the tracking error given in (3) can be readily assessed using either Lyapunov stability theory or Popov's absolute stability theory.

Indeed, the above facts have enlarged the flexibility of selecting and developing optimization algorithms for a given problem and can enormously help for structured optimization where the algorithm structure can be imposed to the optimization a prior. For example, one can select a PID-based optimization algorithm to first close the loop for a given optimization problem as shown in Figure 2, then the PID gains can be regarded as a group of "learning rates" to be selected to guarantee the stability (convergence) of the optimization algorithm and the best tracking performance for the tracking error in (3).

For the gradient descent algorithm in (5), if we denote $z^{-1}$ as the unit delay operator as in the discrete-time control, then the functionality of the "controller" is given by

$$
x_{k}=\frac{-\theta}{1-z^{-1}} \frac{\partial J\left(x_{k-1}\right)}{\partial x_{k-1}}
$$

If a PI structure is used with the performance function as a direct feedback signal, we have

$$
\begin{gathered}
x_{k}=K_{p}\left(0-J\left(x_{k-1}\right)\right)+K_{I} \rho_{k} \\
\rho_{k}=\rho_{k-1}+h\left(0-J\left(x_{k-1}\right)\right)
\end{gathered}
$$

where $h>0$ is the sampling length, and $\left\{K_{p}, K_{I}\right\}$ are the "PI control" gains to be selected so that the closed loop control system shown in Figure 2 is stable and the closed loop system has a good tracking performance albeit $J$ does not always approach the zero set-point. We can also control the gradient by using $\frac{\partial J\left(x_{k-1}\right)}{\partial x_{k-1}}$ as the feedback signal to the controller with a PI structure, in this case the following optimization algorithm can be formulated 


$$
\begin{gathered}
x_{k}=K_{p}\left(0-\frac{\partial J\left(x_{k-1}\right)}{\partial x_{k-1}}\right)+K_{I} \rho_{k} \\
\rho_{k}=\rho_{k-1}+h\left(0-\frac{\partial J\left(x_{k-1}\right)}{\partial x_{k-1}}\right)
\end{gathered}
$$

In comparison with the direct performance function feedback, the above gradient feedback aims at achieving a zero gradient when $k \rightarrow+\infty$ as seen in the integration functionality.

Since in general $J(x)$ is a static nonlinear function and the optimization algorithm is in a recursive ('dynamic') form, the search for a proper optimization algorithm would mean that one need to select a dynamic "controller" that can control a static nonlinear plant well. This has further indicated that optimization is indeed a sub-problem of closed loop control system design.

\subsection{Source of uncertainties in decision making - system uncertainties vs human behavior uncertainties}

For complex industrial processes, human operators at different layer in Figure 1 generally participate in the optimization or decision making. For example, in the operation control layer the operators can be involved in the tuning of the set-points to the control loops in an intuitive way using their operational experience. In this decision making phase there are certain uncertainties involved. For instance, an experienced operator would exhibit less uncertainties in his/her reasoning when making the decision. Such uncertainties can be represented as a disturbance to the "controller" in Figure 3. Of course the "plant" is generally subjected to some degree of uncertainties in terms of the uncertainties embedded in both the performance function and the constraints. Indeed, the effect of uncertainties to the plant has been well studied and is generally treated as a robust optimization problem, where the objective of the optimization is to select a proper set of decision variables $x$ so that the performance index $J$ is minimized when it is subjected to uncertainties.

In this context, the realistic optimization problem can be further represented as in Figure 4, where two types of uncertainties - one to the "controller" and the other to the "plant" are included. The optimization problem can therefore be generalized as to solve the following problem,

$$
\begin{aligned}
& \min _{x} J(x, w) \\
& \text { s.t. } f(x, v)=0
\end{aligned}
$$

where $w$ and $v$ are the uncertainties embedded in the performance function and the constraints and are assumed to be independent random processes whose probability density functions are used to represent their characteristics (see Figure 2). The purpose of the optimization is therefore to select a good $x$ so that $J$ is made to follow the zero set-point as close as possible in the presence of $w$ and $v$, and the uncertainties in the "controller".

In comparison with standard controller design, the uncertainties embedded in the controller is a new phenomenon as in general for the closed loop control design and implementation there is no uncertainties for the controller. This presents a new challenge where uncertainties such as the randomness of human

decision making need to be considered in the optimization phase. Indeed, when the constraint is given by, 


$$
f(x, v)=f(x+v)=0
$$

then $v$ would represent the uncertainties and randomness in the human decision making phase whose pdfs can be obtained using EEG signal as shown in Figure 2.

Since the uncertainties in the decision making phase of human operators can be characterized using the signals from BCI, they can be quantitatively represented as a set of random processes which can be eventually expressed by the pdfs of the BCI signals after the subtraction of the signals themselves from their mean values. In this context, the following generalized structure can be obtained which presents a framework of optimization when it is subjected to uncertainties from both the process and the optimization algorithms.

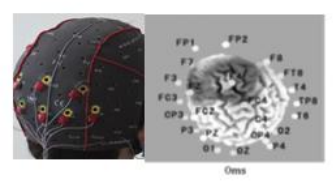

Uncertainties of human decision making
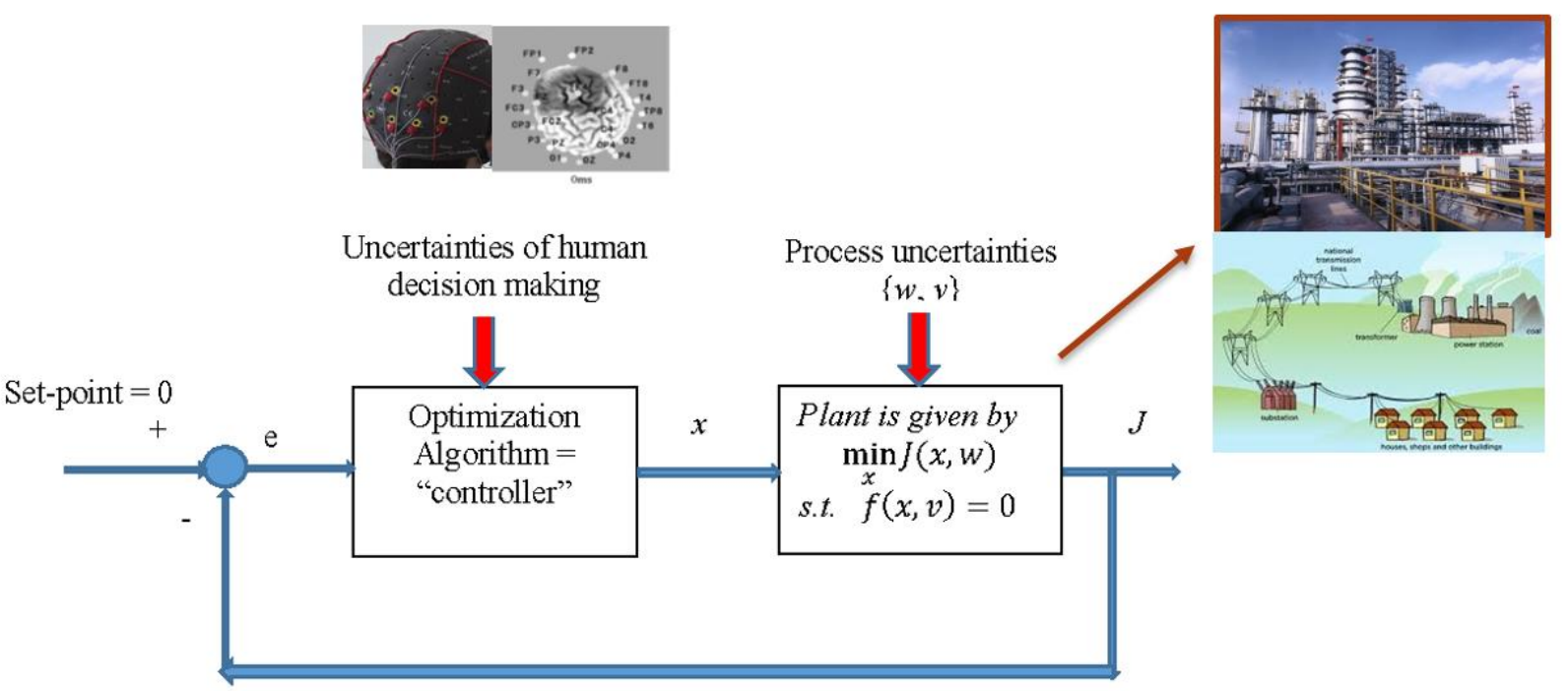

Figure 4. The generalized optimization framework that incorporates uncertainties from both the process and the human decision making phase

This framework reveals that the actual task of the optimization should be to select a "good" $x$ so that $J$ is made to be as close as possible to zero when the closed loop system is subjected to uncertainties both in the "controller" and in the "plant", whilst ensuring the closed loop stability and robustness. 


\subsection{The Generalized Framework for Decision Making Using Probability Density Function (pdf) Shaping Approach}

Since the process performance is subjected to the uncertainties as represented by $w$ in equations (4), $J$ is a random process and its optimization should be performed so that the mean value of $J$ is minimized whilst the randomness of the optimized $J$ is minimized. This is a pdf shaping problem for $J$ where the stochastic distribution control theory originated by Wang ([7], [11] - [19]) can be readily applied so that the pdf of $J$ is moved as close as possible to the left and its spread area is made as narrow as possible. This is shown in Figure. 5.

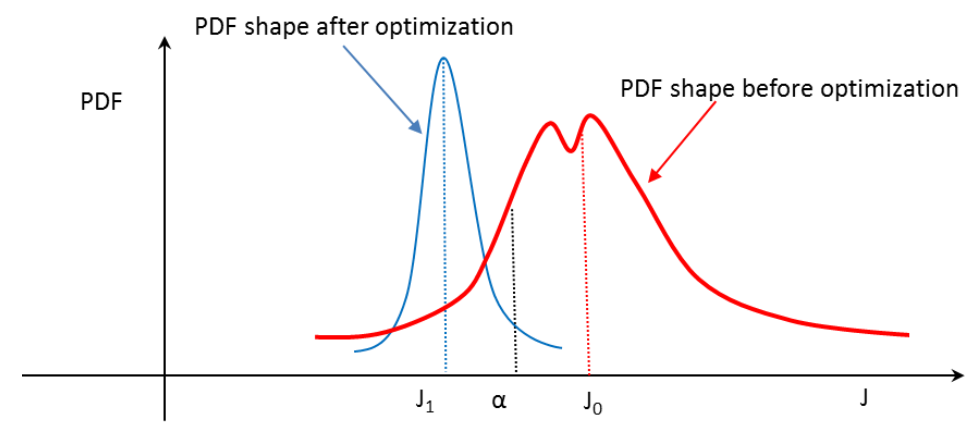

Figure 5. PDFs of the performance function before and after the optimization

\subsection{PDF shaping for the performance function}

Denote the pdf of $J$ as $\gamma_{J}(x, \tau)$ where $\tau \in[a, b]$ is the definition variable that defines $\gamma_{J}(x, \tau), a$ and $b$ are two positive known numbers that stands for the definition interval, then to realize the above purpose of optimization we need to minimize the following induced functional distance

$$
\pi_{0}(x)=\left\|\delta(\tau-a)-\gamma_{J}(x, \tau)\right\|=\sqrt{\int_{a}^{b}\left[\delta(\tau-a)-\gamma_{J}(x, \tau)\right]^{2} d \tau}=\min
$$

subjected to the constraint expressed in (5), where $\delta(\tau-a)$ is an impulse pdf function defined as follows:

$$
\delta(\tau-a)=\left\{\begin{array}{l}
+\infty, \quad \tau=a \\
0, \text { otherwise }
\end{array}\right.
$$

Without loss of generality, one can just minimize the following subjected to constraint (5).

$$
\pi(x)=\int_{a}^{b}\left[\delta(\tau-a)-\gamma_{J}(x, \tau)\right]^{2} d \tau=\min
$$

This presents a much generalized approach for stochastic optimization where the existing rather rich results on mean value and variance based stochastic optimization become a special case of (7). 


\subsection{Dealing with the constraint}

Whilst solving (7) is a straightforward stochastic distribution control problem, dealing with the constraint $f(x, v)=0$ at the same time is not easy as $v$ is a random process. Satisfying such a constraint would mean that $x$ should be a random variable applied to the actual process. Here we try to transfer this constraint into another pdf shaping problem albeit in general we can use the mean value of the optimized $x$ as the actual decision variable to be applied to the plant (see the next section).

For this purpose, we denote the pdf of $f(x, v)$ as $\gamma_{f}(x, \varphi)$ where $\varphi \in[c, d]$ is a definition variable for $\gamma_{f}(x, \varphi)$ with known $c<0$ and $d>0$, then to realize $f(x, v)=0$, we need to make sure that the following

$$
\gamma_{f}(x, \varphi)=\delta(\varphi), \quad \forall \varphi \in[c, d]
$$

is satisfied for the selected optimal $x$. This means that we need to make sure that the pdf of $f(x, v)$ equals to a $\delta$ - function, which can be realized "somehow" by minimizing the following functional distance by selecting a good decision variable $x$

$$
\varepsilon_{0}(x)=|| \delta(\varphi)-\gamma_{f}(x, \varphi) \|=\sqrt{\int_{c}^{d}\left[\delta(\varphi)-\gamma_{f}(x, \varphi)\right]^{2} d \varphi}
$$

or to simply minimize the following

$$
\varepsilon(x)=\int_{c}^{d}\left[\delta(\varphi)-\gamma_{f}(x, \varphi)\right]^{2} d \varphi
$$

As a result, the actual optimization becomes finding $x$ so that both $\pi(x)$ and $\varepsilon(x)$ are minimized at least and at the same time. Let $x_{k}(k=1,2, \ldots)$ be the sequence of the decision variable at sample number $k$, then to obtain the optimal $x_{o p t}$ that optimizes $J$ subjected to $f(x, v)=0$, we need to select $x_{k}$ so that the following performance function is minimized.

$$
J_{\sigma}=\pi\left(x_{k}\right)+\sum_{j=1}^{k} \varepsilon\left(x_{j}\right), \quad k=1,2,3, \ldots
$$

If the above can be minimized (i.e., a sufficient condition) for $k$ going to infinite, the constraint $f(x, v)=0$ can be strictly guaranteed. This is because when $k$ goes to infinite we have

which indicates that

$$
\sum_{j=1}^{+\infty} \varepsilon\left(x_{j}\right)<+\infty
$$

$$
\begin{gathered}
\lim _{k \rightarrow+\infty} \varepsilon\left(x_{k}\right)=\lim _{k \rightarrow+\infty} \int_{c}^{d}\left[\delta(\varphi)-\gamma_{f}\left(x_{k}, \varphi\right)\right]^{2} d \varphi=0 \\
\lim _{k \rightarrow+\infty} \gamma_{f}\left(x_{k}, \varphi\right)=\delta(\varphi), \quad \forall \varphi \in[c, d]
\end{gathered}
$$

As a result, the optimal decision variable can be made to converge by solving (10) which gives 


$$
\lim _{k \rightarrow+\infty} x_{k}=x_{o p t}
$$

This leads to the following theorem that states a sufficient condition for solving the stochastic optimization problem given in (4) - (5).

THEOREM 1 (The Discrete-time Case). A sufficient condition for solving the optimization problem (4) -(5) is that there is a sequence of decision variable $\left\{x_{k}\right\}$ for $k=1,2,3, \ldots$ so that the following inequality $\underline{\text { holds }}$

$$
\sum_{k=1}^{+\infty} \varepsilon\left(x_{k}\right)<+\infty
$$

where it has been denoted that

$$
\varepsilon\left(x_{k}\right)=\int_{c}^{d}\left[\delta(\varphi)-\gamma_{f}\left(x_{k}, \varphi\right)\right]^{2} d \varphi
$$

When $\Delta x=x_{k}-x_{k-1}$ is very small, a continuous-time format can be obtained for (10) to give

$$
J_{\sigma}(x)=\pi\left(x_{t}\right)+\int_{0}^{t} \int_{c}^{d}\left[\delta(\varphi)-\gamma_{f}(x(\tau), \varphi)\right]^{2} d \varphi d \tau
$$

In this case we need to find a profile $x(\tau)$ for $\tau \in[0, t]$ that can minimize (14) for any $t>0$, this means that we need to solve the following optimization problem

$$
\min _{x(\tau), \tau \in[0, t]}\left\{\pi\left(x_{t}\right)+\int_{0}^{t} \int_{c}^{d}\left[\delta(\varphi)-\gamma_{f}(x(\tau), \varphi)\right]^{2} d \varphi d \tau\right\} \quad \forall t \in[0,+\infty)
$$

This is an optimization problem, if it is solvable for any $t>0$, then it can be concluded that

which indicates that

$$
\int_{0}^{t} \int_{c}^{d}\left[\delta(\varphi)-\gamma_{f}(x(\tau), \varphi)\right]^{2} d \varphi d \tau<+\infty, \text { for } \forall t>0
$$

$$
\lim _{t \rightarrow+\infty} \int_{c}^{d}\left[\delta(\varphi)-\gamma_{f}(x(t), \varphi)\right]^{2} d \varphi=0
$$

As a result, it can be obtained that

$$
\begin{gathered}
\lim _{t \rightarrow+\infty} \gamma_{f}(x(t), \varphi)=\delta(\varphi), \quad \text { for } \forall \varphi \in[c, d] \\
\lim _{t \rightarrow+\infty} x(t)=x_{\text {opt }}
\end{gathered}
$$

Along with the above formulation, the following theorem can also be readily obtained for the continuoustime case. 
THEOREM 2 (The Continuous-time Case). A sufficient condition for solving the optimization problem (4)-(5) is that there is a function $x(\tau)$ for $\tau \in[0, t]$ so that the following inequality holds

$$
\int_{0}^{t} \int_{c}^{d}\left[\delta(\varphi)-\gamma_{f}(x(\tau), \varphi)\right]^{2} d \varphi d \tau<+\infty, \text { for } \forall t>0
$$

\subsection{A total probabilistic solution}

Since constraint (5) indicates that in general $x$ is also a random variable, another way to solve (4)-(5) is to find an optimal probability density function for the decision variable $x$ so that the probability density function of the performance function $J$ is made as left and as narrow as possible. Denote the optimal probability density function of $x$ as $\gamma_{x}(\tau), \tau \in[e, f]$ for a known $e$ and $f$, then with this pdf one can find a value of $x$ to apply to the system. For example, the optimal and applicable $x_{\text {opt }}$ can be calculated from

$$
x_{o p t} \approx \int_{e}^{f} \tau \gamma_{x}(\tau) d \tau
$$

Since such as an approach requires the optimal solution of the pdf of $x$, it is referred to as the total probabilistic solution for (4)-(5). Note that equation (5) is only an approximate as in practice $\gamma_{x}(\tau)$ cannot be directly applied to the system. This constitutes a marked disadvantage of this type of solution.

\subsection{Relations to chance constrained optimization}

Problem (4)-(5) can also be regarded as a chance constrained optimization problem ([9] - [10]) studied in optimal power flow design in the sense that we need to find an optimal $x$ for the following problem.

$$
\begin{aligned}
& \min _{x} J(x, w) \\
& \text { s.t. } \operatorname{Prob}\{f(x, v)=0\} \geq 1-\beta
\end{aligned}
$$

where $\beta \approx 0$ is a very small positive number. The above can be further relaxed into the following problem

$$
\begin{aligned}
& \min _{x} E_{w}\{J(x, w)\} \\
& \text { s.t. } \operatorname{Prob}\{f(x, v) \leq 0\} \geq 1-\beta=\alpha
\end{aligned}
$$

where $E_{w}\{$.$\} is the mathematical expectation operator over random variable w$. Indeed, this is a standard chance constrained optimization problem. In pdf shaping sense, this means that we need to find a good $x$ so that the pdf of $J$ is moved as left and as narrow as possible together with the pdf of $f(x, v)$. As a result, the formulation and sufficient conditions stated in Theorems 1 and 2 can all be applied to seek the solutions to the above chance constrained optimization problem.

Of course, in this context the rather rich algorithms developed in stochastic distribution control ([7] - [8]) can be used to solve chance constrained optimization. For example, using the B-spline expression for $\gamma_{f}(x, \varphi)$, we have 


$$
\gamma_{f}(x, \varphi)=\sum_{i=1}^{N} \theta_{i}(x) B_{i}(\varphi), \quad \forall \varphi \in[c, d]
$$

where $B_{i}(\varphi),\{i=1,2, \ldots, N\}$ are the set of pre-specified basis functions defined on $[c, d]$, and $\theta_{i}(x),\{i=$ $1,2, \ldots, N\}$ are B-spline expansion weights that are related to $x$. Then the probability constraint (19) is equivalent to

$$
\int_{c}^{0} \gamma_{f}(x, \varphi) d \varphi=\sum_{i=1}^{N} \theta_{i}(x) \int_{c}^{0} B_{i}(\varphi) d \varphi \geq \alpha
$$

If we denote $\gamma_{w}(\tau)$ as the pdf of $w$ where without loss of generality it has assumed that $\tau \in[a, b]$, then it can be obtained that

$$
E_{w}\{J(x, w)\}=\int_{a}^{b} J(x, \tau) \gamma_{w}(\tau) d \tau=J_{1}(x)
$$

In this case the chance constrained optimization (18) - (19) can be transferred into the solving of the following deterministic nonlinear optimization problem

$$
\begin{array}{ll} 
& \min _{x} J_{1}(x) \\
\text { s.t. } & \sum_{i=1}^{N} \theta_{i}(x) \int_{c}^{0} B_{i}(\varphi) d \varphi \geq \alpha
\end{array}
$$

\subsection{A case study in economic power dispatch}

\subsection{Case description}

In this section, we will apply the proposed pdf-shaping optimization approach to power system dispatch with intermittent generation sources such as wind farms, etc. Assume that we have the widely used IEEE 3-machine 9-bus system shown in Figure 6, and that there is a wind farm connected to bus 7. In this case, we will consider the uncertainties of the wind energy and load demand. 


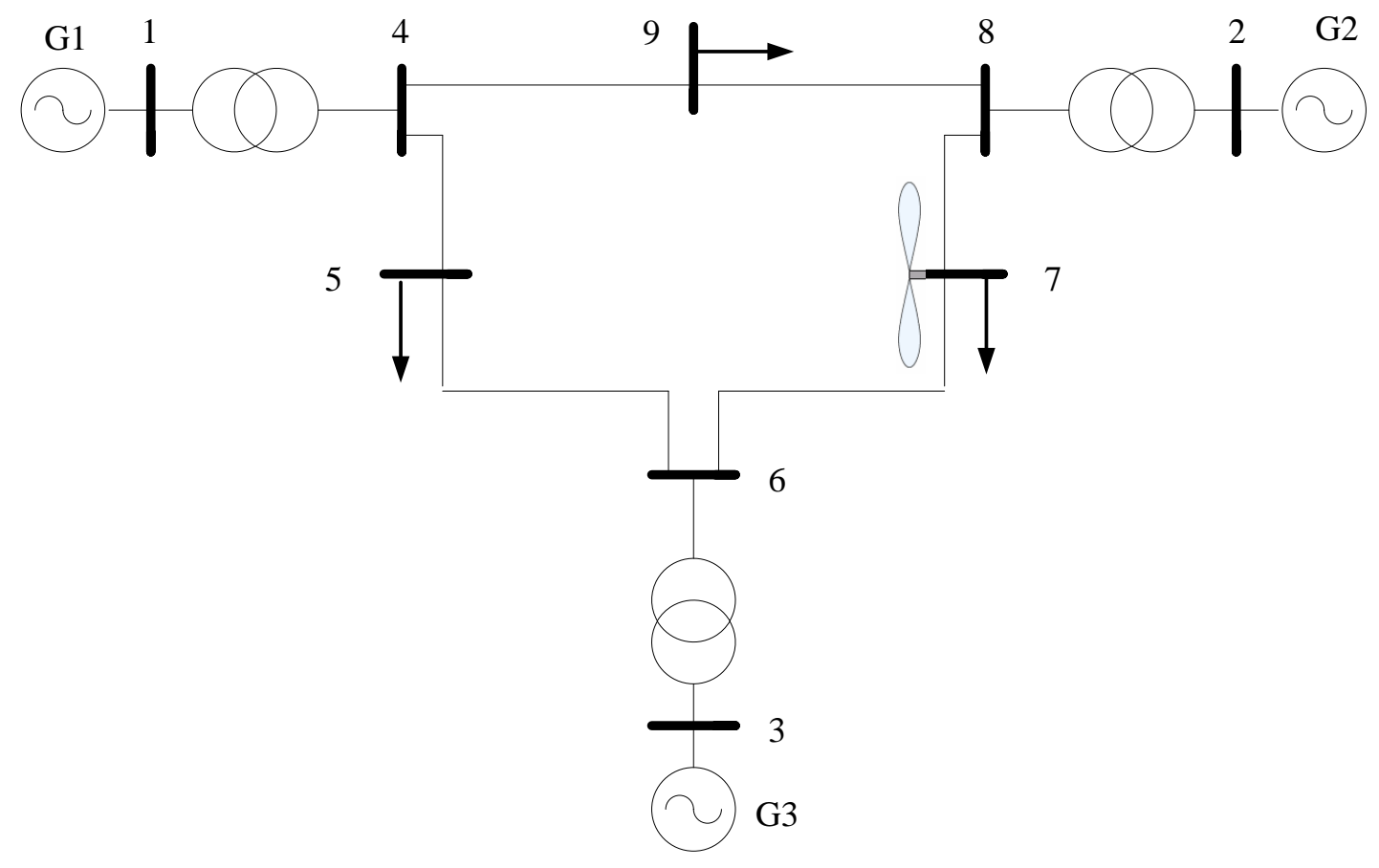

Figure 6. Case study: the IEEE 9-bus test system

The objective of this case is to minimize both the mean and variance of the total generation cost, and it is donated as

$$
\min [E(J)+D(J)]
$$

where the performance function is defined as

$$
J=\sum_{t=1}^{T} \sum_{i=1}^{N} U_{i t} f_{i t}\left(P_{i t}\right)
$$

In equations (25) - (26), E(J) is the mean value of $J ; D(J)$ is the variance of $J ; T$ is the number of hours; $N$ is the number of generators; $U_{i t}$ is the status of generators ( $U_{i t}=1$ means that generator $i$ is in use in time slot $t ; U_{i t}=0$ indicates that generator $i$ is out of use in time slot $\left.t\right)$; and $f_{i t}\left(P_{i t}\right)$ is the cost function of generators shown as follows.

$$
f_{i t}\left(P_{i t}\right)=a_{i}+b_{i} P_{i t}+c_{i} P_{i t}^{2}
$$

Here $a_{i}, b_{i}, c_{i}, d_{i}$ and $e_{i}$ are pre-specified constants; $P_{i t}^{\min }$ is the lower limit of the generator output in time slot $t$.

The actual optimization are subjected to the following constraints:

1. Power balance constraint 


$$
\sum_{i=1}^{N} P_{i t}+\sum_{j=1}^{M} P_{i t}^{W}-P_{D t}-P_{L t}=0, \quad t \in T
$$

Here $M$ is the number of wind plants; $P_{i t}^{W}$ is the output of wind plant in time slot $t ; P_{D t}$ is the load in time slot $t ; P_{L t}$ is loss in time slot $t$. Here $P_{i t}^{W}$ and $P_{D t}$ are stochastic variables. This type of constraints are in line with constraint $f(x, v)=f(x+v)=0$, where the human decision uncertainties can be grouped together with $P_{i t}^{W}$ or $P_{D t}$.

2. Output of generators constraint

$$
P_{i t}^{\min } \leq P_{i t} \leq P_{i t}^{\max }, \quad i \in N, t \in T
$$

Here $P_{i t}^{\max }$ is the limit of the generator output in time slot $t$.

3. Ramp rate constraint

$$
P\left\{D_{R i} \leq P_{i t}-P_{i(t-1)} \leq U_{R i}\right\} \geq \beta_{1}, \quad i \in N, t \in T
$$

Here $D_{R i}$ is the ramp down rate; $U_{R i}$ is the ramp up rate; $\beta_{1}$ is the confidence level.

4. Spinning reserve constraint

$$
\begin{aligned}
& P\left\{\sum_{i=1}^{N}\left(P_{i t}^{\max }-P_{i t}\right) \geq U_{S R t}\right\} \geq \beta_{2}, \quad i \in N, t \in T \\
& P\left\{\sum_{i=1}^{N}\left(P_{i t}-P_{i t}{ }^{\min }\right) \geq D_{S R t}\right\} \geq \beta_{3}, \quad i \in N, t \in T
\end{aligned}
$$

Here $D_{S R t}$ is the low limit of spinning reserve; $U_{S R t}$ is the up limit of spinning reserve; $\beta_{2}$ and $\beta_{3}$ are confidence levels.

Assume that all the generators are on operation, namely, $U_{i t}=1$ and $\mathrm{N}=24$. In constraints, by neglecting the Spinning reserve constraint and Ramp rate constraint, finally we have the following optimization problem:

$$
\begin{aligned}
& \min [E(J)+D(J)] \\
& \text { s.t. } \\
& \sum_{i=1}^{3} P_{i t}+P_{t}^{W}-P_{D t}-P_{L t}=0 \\
& P_{i t}^{\min } \leq P_{i t} \leq P_{i t}^{\max }
\end{aligned}
$$

Here $J=\sum_{t=1}^{24} \sum_{i=1}^{3} f_{i t}\left(P_{i t}\right) ; P_{i t}{ }^{W}$ is the wind energy, $P_{D t}$ is the load and they are both stochastic variables. Denote $P_{n}=-\left(P_{t}^{W}-P_{D t}\right)$ is a stochastic variable with mean $\bar{P}_{n}$ and variance $\Delta P_{n}$.

Then the hourly cost function can be given by 


$$
\begin{aligned}
& J=f_{1}\left(P_{1}\right)+f_{2}\left(P_{2}\right)+f_{3}\left(P_{3}\right) \\
& \quad=a_{1}+b_{1} P_{1}+c_{1} P_{1}^{2}+a_{2}+b_{2} P_{2}+c_{2} P_{2}^{2}+a_{3}+b_{3} P_{3}+c_{3} P_{3}^{2} \\
& \text { s.t. } \\
& P_{1}+P_{2}+P_{3}-P_{n}=0 ; \\
& P_{i t}^{\min } \leq P_{i t} \leq P_{i t}^{\max }
\end{aligned}
$$

Assume that the pdf of $P_{n}$ is of a normal distribution and known as $N\left(\mu_{n}, \sigma_{n}^{2}\right) . P_{1}$ is subjected to a normal distribution of $N\left(\mu_{1}, \sigma_{1}^{2}\right) ; P_{2}$ belongs to a normal distribution of $N\left(\mu_{2}, \sigma_{2}^{2}\right)$; and $P_{3}$ belongs to a normal distribution of $N\left(\mu_{n}-\mu_{1}-\mu_{2}, \sigma_{n}^{2}-\sigma_{1}^{2}-\sigma_{2}^{2}\right) ; P_{1}$ and $P_{2}$ are independent. We have the mean of the cost function $J$ calculated as follows:

$$
\begin{aligned}
\mu(J) & =f_{1}\left(\mu_{1}\right)+f_{2}\left(\mu_{2}\right)+f_{3}\left(\mu_{3}\right)+f^{\prime \prime}{ }_{1}\left(\mu_{1}\right) \sigma_{1}^{2}+f^{\prime \prime}{ }_{2}\left(\mu_{2}\right) \sigma_{2}^{2}+f^{\prime \prime}{ }_{3}\left(\mu_{3}\right) \sigma_{3}^{2} \\
& =a_{1}+b_{1} \mu_{1}+c_{1} \mu_{1}^{2}+a_{2}+b_{2} \mu_{2}+c_{2} \mu_{2}^{2}+a_{3}+b_{3} \mu_{3}+c_{3} \mu_{3}^{2}+c_{1} \sigma_{1}^{2}+c_{2} \sigma_{2}^{2}+c_{3} \sigma_{3}^{2}
\end{aligned}
$$

The variance of the cost function can also be calculated to read

$$
\begin{aligned}
D(J) & \approx f_{1}^{\prime}\left(\mu_{1}\right) \sigma_{1}^{2}+f_{2}^{\prime}\left(\mu_{2}\right) \sigma_{2}^{2}+f_{3}^{\prime}\left(\mu_{3}\right) \sigma_{3}^{2} \\
& \approx\left(b_{1}+2 c_{1} \mu_{1}\right)^{2} \sigma_{1}^{2}+\left(b_{2}+2 c_{2} \mu_{2}\right)^{2} \sigma_{2}^{2}+\left(b_{3}+2 c_{3} \mu_{3}\right)^{2} \sigma_{3}^{2}
\end{aligned}
$$

The pdf-shaping method requires to minimize both the expectation and variance of the objective function $J$, which means that we need to solve the following for the optimization

$$
\left\{\begin{array}{l}
\frac{\partial \mu(J)}{\partial X}=0 \\
\frac{\partial D(J)}{\partial X}=0
\end{array}\right.
$$

where $X=\left[\mu_{1}, \mu_{2}, \sigma_{1}^{2}, \sigma_{2}^{2}\right]$. The optimal $X$ can be determined by solving (37).

\subsection{Results and economic profitability analysis}

The results of pdf-shaping method on the stochastic economic dispatch is shown in Figure 7. The red-bar curve is the generation cost before optimization, while the green line represents the cost after PDF-shaping. Note that both the mean value and variance are significantly reduced. The mean cost is reduced from 2204 to 1847 , while the variance is reduced from 89 to 50 . 


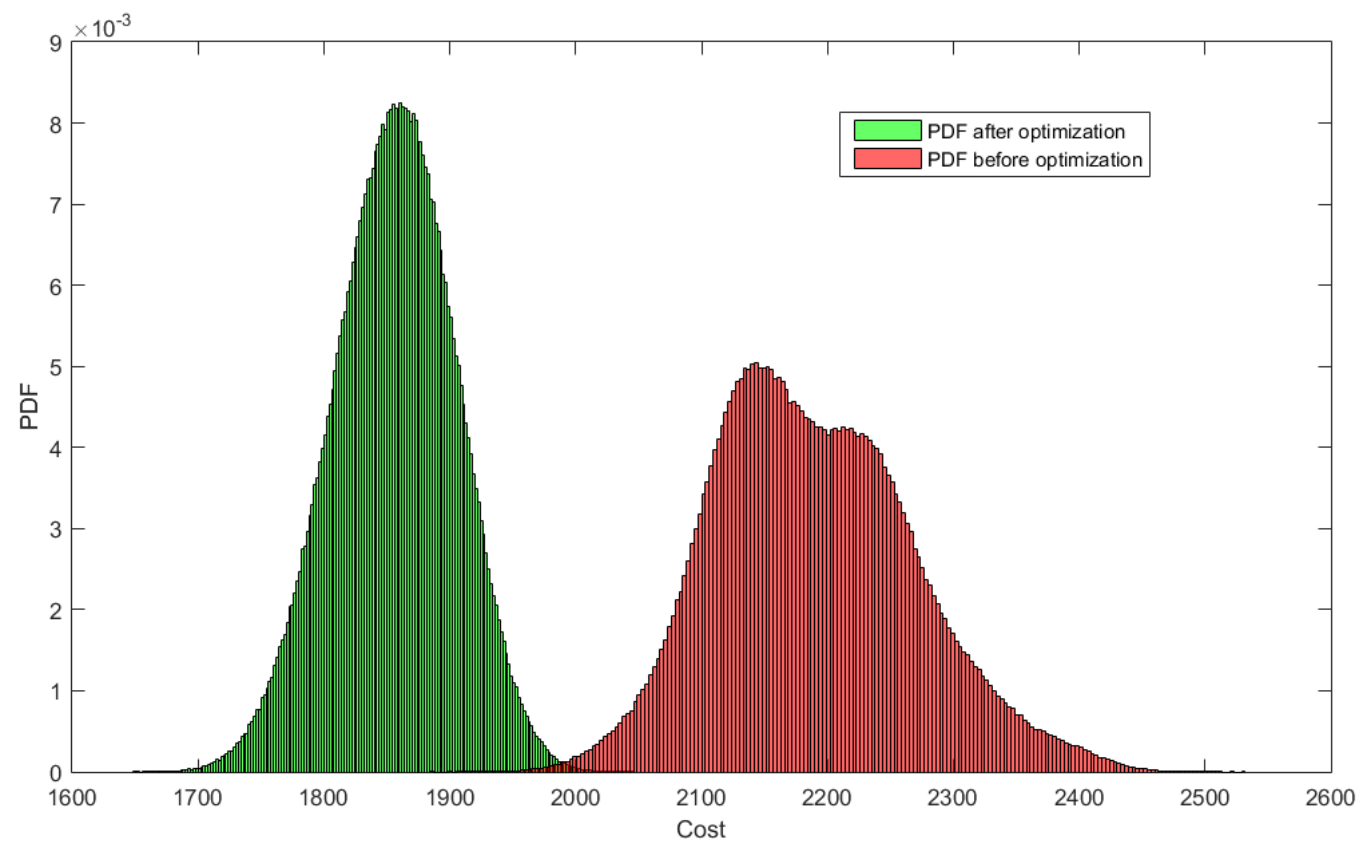

Figure 7. PDF-Shaping results on stochastic economic dispatch

We have obtained the results as follows. Figure 8 shows the optimization results for the comparison of single objective and multi-objective. As it can be seen from Figure 8, the traditional single objective optimization results in the long tail pdf phenomenon which means a significant risk of high cost shown in Figure 9.

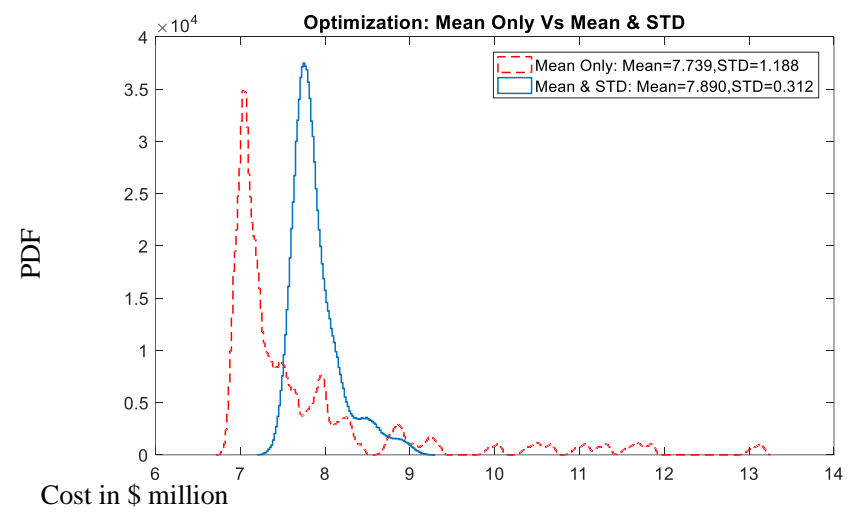

Fig. 8. Optimization results of PDF: single objective VS multi-objective

It can be seen from Figure 5 that the probability of single objective optimization for cost being greater than 9 million dollars is $15 \%$, namely,

$$
P\{\text { COST }>\$ 9 \text { million }\}=15 \% \text {. }
$$

However, the proposed multi-objective optimization is able to reduce the probability to $0.5 \%$, namely,

$$
P\{\text { COST }>\$ 9 \text { million }\}=0.5 \% \text {. }
$$


This mean that a huge saving can be expected in practice.

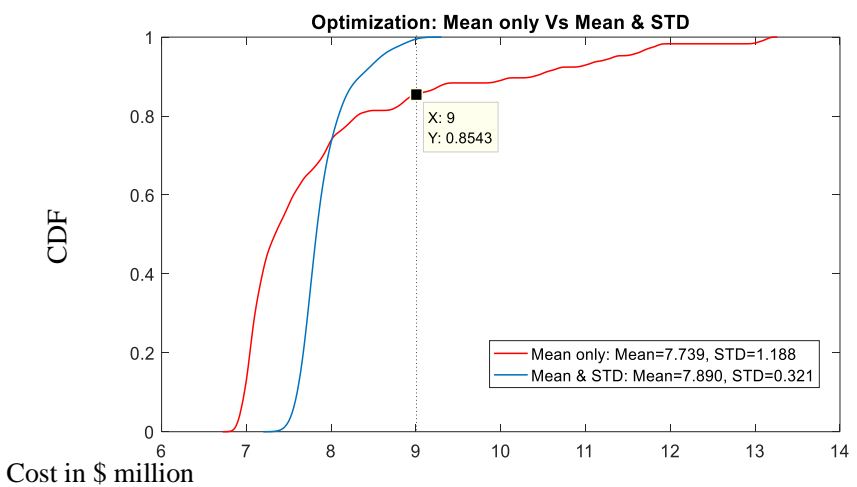

Fig. 9. Optimization of CDF: single objective VS multi-objective

\subsection{Switching strategy for whole horizon economic power dispatch}

Of course, it can be seen from Figure 9 that if the cost boundary is below $\$ 7.8 \mathrm{M}$, single optimization gives better result. This indicates that further improvement can be made to the proposed algorithm. For example, with a good load forecasting one can determine whether the cost would be less or larger than $\$ 7.8 \mathrm{M}$ threshold. If it is less than $\$ 7.8 \mathrm{M}$ then we should only use mean value minimization, otherwise we should use both mean and variance based minimization. This indicates that a switching function can be added to the proposed pdf shaping based optimization algorithm.

\subsection{Conclusions}

In this project, a general framework has been obtained which shows that

(1) The optimization is a special case of closed loop control system design where rather rich control theory can be directly applied to deal with optimization issues.

(2) Uncertainties of human operator's decision making phase have been characterized and embedded into the stochastic optimization scenario in a closed loop framework.

(3) A sufficient condition on pdf shaping based stochastic optimization has been derived.

(4) Encouraging results have been obtained for the economic power dispatch where a huge saving on generation cost can be expected. 


\subsection{References}

[1] Adetola, V., \& Guay, M. (2010). Integration of real-time optimization and model predictive control. Journal of Process Control, 20, 125-133.

[2] Darby, M. L., Nikolaou, M., Jones, J., \& Nicholson, D. (2011). RTO: An overview and assessment of current practice. Journal of Process Control, 21, 874-884.

[3] Zhang, C., Wang, H., \& Wu, M. H. (2013). EEG-based expert system using complexity measures and probability density function control in alpha sub-band. Integrated computer-aided engineering, 20(4), 391405.

[4] Kehoe, B, Patil, S., Abbeel, P. and Goldberg, K. (2015). A survey of research on cloud robotics and automation. IEEE Transactions on Automation Science and Engineering: Special Issue on Cloud Robotics and Automation. Vol. 12, no. 2. Apr. 2015.

[5] Mason S.G., \& Birch. G.E. (2000). A Brain-Controlled Switch for Asynchronous Control Applications, IEEE Trans. Biomedical Engineering, 47(10), 1297-1307.

[6] Wairagkar, M., Daly, I., Hayashi, Y. \& Nasuto, S. J. (2016). Autocorrelation based EEG Dynamics depicting Motor Intention, in Proceedings of the 6th International Brain-Computer Interface Meeting, organized by the BCI Society, 2016.

[7] Wang (1999), H, Bounded Dynamic Stochastic Distributions - Modelling and Control, Springer-Verlag, London (book publication).

[8] Wang, H., \& Afshar, P. (2009). ILC-based fixed-structure controller design for output PDF shaping in stochastic systems using LMI techniques, IEEE Transactions on Automatic Control, Vol. 54, No. 4, pp. $760-773,$.

[9] Zhang, H., \& Li, P. (2011). Chance constrained programming for optimal power flow under uncertainty, IEEE Transactions on Power Systems, Vol. 26, No. 4, pp. 2417 - 2424.

[10] Geletu, A., \& Li, P. (2014). Recent developments in computational approaches to optimization under uncertainty and application in process systems engineering, ChemBioEng Reviews, pp. 170 - 190.

[11] L. Guo, and H. Wang., Stochastic Distribution Control Systems Design: A Convex Optimization Approach (book publication), total 191 pages, Springer-Verlag, London, April, 2010.

[12] M. F. Ren, J. H. Zhang and H. Wang, non-Gaussian System Control and Filtering, Chinese Science Publishers, July, 2016 (book publication)

[13] H. Wang, "Robust control of the output probability density functions for multivariable stochastic systems with guaranteed stability", IEEE Transactions on Automatic Control, Vol. 41, pp.2103-2107, 1999.

[14] H. Wang, J. H. Zhang, "Bounded stochastic distribution control for pseudo ARMAX systems", IEEE Transactions on Automatic Control, Vol.46, pp.486-490, 2001. 
[15] H. Wang, "Minimum entropy control for non-Gaussian dynamic stochastic systems", IEEE Transactions on Automatic Control, Vol. 47, pp.398 - 403, 2002.

[17] Y. Liu, H. Wang and L. Guo, Observer-based feedback controller design for a class of stochastic systems with non-Gaussian variables, IEEE Transactions on Automatic Control, No. 5, pp. 1445 - 1450, 2015.

[18] J. L. Zhou, X. Wang, J. F. Zhang, H. Wang and G. H. Yang, A new minimum entropy controller design and the control loop performance assessment for output stochastic distribution systems, IEEE Transactions on Automatic Control, Vol. 60, pp. 2524 - 2529, 2015, . .

[19] M Ren, J Zhang and H Wang, Minimized tracking error randomness control for nonlinear multivariate and non-Gaussian systems using the generalized density evolution equation, IEEE Transactions on Automatic Control, VOL. 59, NO. 9, pp. 2486 - 2490, 2014,. 


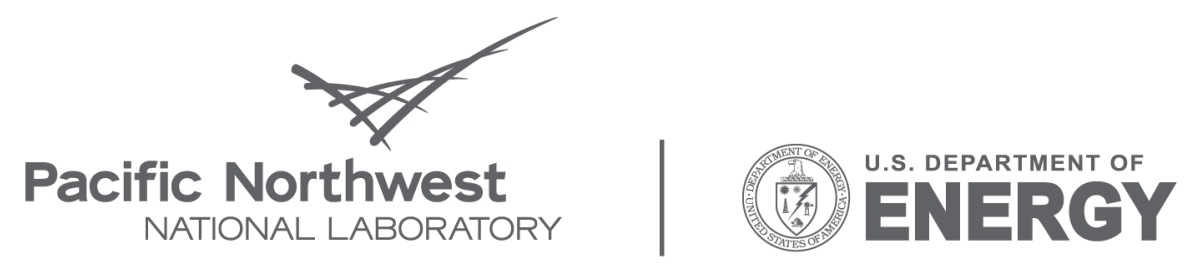

Proudly Operated by Battelle Since 1965

902 Battelle Boulevard

P.O. Box 999

Richland, WA 99352

1-888-375-PNNL (7665)

www.pnl.gov 